\title{
Article \\ Ursolic Acid Protects Sodium Dodecyl Sulfate-Induced Drosophila Ulcerative Colitis Model by Inhibiting the JNK Signaling
}

\author{
Tian Wei ${ }^{1,2,+}$, Lei $\mathrm{Wu}^{1,+}$, Xiaowen Ji ${ }^{1}$, Yan Gao ${ }^{1}$ and Guiran Xiao ${ }^{1, *(D)}$ \\ 1 Key Laboratory of Metabolism and Regulation for Major Diseases of Anhui Higher Education Institutes, \\ School of Food and Biological Engineering, Hefei University of Technology, Hefei 230009, China; \\ weitfield@126.com (T.W.); 18605666360@163.com (L.W.); jxwjyl@163.com (X.J.); 13095531206@163.com (Y.G.) \\ 2 Department of Toxicology, School of Public Health, Anhui Medical University, Hefei 230032, China \\ * Correspondence: xiaoguiran@hfut.edu.cn; Tel.: +86-177-3022-7689 \\ + These authors contributed equally to this work.
}

Citation: Wei, T.; Wu, L.; Ji, X.; Gao, Y.; Xiao, G. Ursolic Acid Protects Sodium Dodecyl Sulfate-Induced Drosophila Ulcerative Colitis Model by Inhibiting the JNK Signaling. Antioxidants 2022, 11, 426. https:// doi.org/10.3390/antiox11020426

Academic Editor: Salvador Máñez Aliño

Received: 15 January 2022

Accepted: 18 February 2022

Published: 21 February 2022

Publisher's Note: MDPI stays neutral with regard to jurisdictional claims in published maps and institutional affiliations.

Copyright: (C) 2022 by the authors. Licensee MDPI, Basel, Switzerland. This article is an open access article distributed under the terms and conditions of the Creative Commons Attribution (CC BY) license (https:// creativecommons.org/licenses/by/ $4.0 /)$.

\begin{abstract}
Ursolic acid (UA) is a bioactive molecule widely distributed in various fruits and vegetables, which was reported to play a therapeutic role in ulcerative colitis (UC) induced by toxic chemicals. However, the underlying mechanism has not been well clarified in vivo. Here, using a Drosophila UC model induced by sodium dodecyl sulfate (SDS), we investigated the defensive effect of UA on intestinal damage. The results showed that UA could significantly protect Drosophila from the damage caused by SDS exposure. Further, UA alleviated the accumulation of reactive oxygen species (ROS) and malondialdehyde (MDA) induced by SDS and upregulated the activities of total superoxide dismutase (T-SOD) and catalase (CAT). Moreover, the proliferation and differentiation of intestine stem cells (ISCs) as well as the excessive activation of the c-Jun N-terminal kinase (JNK)-dependent JAK/STAT signaling pathway induced by SDS were restored by UA. In conclusion, UA prevents intestine injury from toxic compounds by reducing the JNK/JAK/STAT signaling pathway. UA may provide a theoretical basis for functional food or natural medicine development.
\end{abstract}

Keywords: ursolic acid; ulcerative colitis; JNK; JAK/STAT; Drosophila

\section{Introduction}

Ulcerative colitis (UC) is a chronic and recurrent inflammatory bowel disease that is listed as one of the modern refractory diseases by the World Health Organization (WHO) [1] UC is characterized with non-specific chronic inflammation of the intestine, which causes various complications, such as increased risk of colorectal cancer [2]. However, some limitations exist in the present therapies, such as unsatisfactory long-term efficacy, drug resistance, and severe systemic side effects $[3,4]$. Therefore, novel effective and sustained treatments are urgently needed. The single-layered simple epithelium of the gastro-intestinal tract controls nutrient uptake, coordinates our metabolism and shields us from luminal components including chemical poison or pathogens $[5,6]$. Therefore, the maintenance of intestinal barrier function, including its metabolic and immune functions, is essential for organismal health. Epithelial homeostasis is dependent on a balance of intestinal stem cell (ISC) selfrenewal, progenitor differentiation, cell shedding and apoptosis [7]. In recent years, more and more studies are focused on how different nutritional states influence ISC function and epithelial homeostasis.

Ursolic acid (UA) is an anti-inflammatory natural triterpenoid found in large quantities in different fruits and vegetables, including apple, basil, cranberry, and peppermint $[8,9]$. Several studies have shown that UA regulates numerous biological processes including antioxidant [10], anti-cancer [11], anti-inflammation [12], anti-bacterial [13] and immunomodulatory processes [7]. UA was recently reported to play a crucial role in intestinal protection $[14,15]$. The chemical compounds sodium dodecyl sulfate (SDS) and dextran 
sulfate sodium (DSS) have previously been shown to induce epithelial cell damage [16]. Therefore, they are widely used to induce UC in many model organisms [17-19]. UA was reported to protect a DSS-induced mouse UC model by reducing the upregulation of NF- $\mathrm{kB}$ in the colon tissues [20]. However, the underlying mechanism of UA restoring UC needs further investigation. New insights have demonstrated that multiple metabolic signaling pathways are involved in the regulation of SDS- or DSS-induced intestinal disruption, such as the c-Jun N-terminal kinase (JNK) [21,22], JAK/STAT, epidermal growth factor receptor (EGFR), and Wnt and Notch pathways [23,24]. UA was previously reported to repress tumor growth through the JNK and JAK/STAT pathways in human cancer cells [17,25], so we wondered whether these pathways are involved in the protection of UA against the intestine damage of UC.

Drosophila melanogaster (hereinafter Drosophila) represents one of the most important model organisms and has made fundamental contributions to different areas of biology [26]. The digestive system of Drosophila contains the foregut, proventriculus, midgut and hindgut, and the intestinal function of Drosophila is similar to that of mammals [27,28]. The Drosophila midgut harbors multipotent adult stem cells that are essential to renew the gut in homeostatic conditions and upon stress-induced regeneration, and the Drosophila midgut has emerged in recent years as a model system to decipher regulatory mechanisms of stem cell biology $[29,30]$. Comparative genomic studies estimate that up to $75 \%$ of the human genes implicated in diseases are conserved in Drosophila [31]. In addition, the signaling pathways involved in development of diseases, such as the JNK, JAK/STAT, Wnt and Notch pathways, are highly conserved from Drosophila to human beings [32,33]. Therefore, Drosophila has become a powerful model to study intestinal physiology and pathology [32] For example, Drosophila has been used to screen plant extracts with potential applications in many chemical-induced intestinal disorders $[34,35]$.

In this study, Drosophila was employed to investigate the effect and regulatory mechanism of UA on the intestine disruption induced by SDS. The results indicated that the decreased survival rate and mobility of adult flies caused by SDS could be restored by UA. In addition, the increased reactive oxygen species (ROS) and malondialdehyde (MDA) levels caused by SDS could be reduced by UA. Further studies indicated that the activated JNK and JAK/STAT pathways induced by ROS in UC models could be rescued by UA. These results elucidate the protective effect of UA on UC caused by environmental stresses. This study is of great significance for UA application and may improve the medicinal and nutritional value of UA in the future.

\section{Materials and Methods}

\subsection{Materials and Reagents}

The UA (purity $\geq 98 \%$ ) was purchased from Aladdin Co., Ltd. (\#U118635, Shanghai, China). The SDS (\#L4509) and Brilliant Blue FCF (\#80717) were purchased from Shanghai Macklin Biochemical Co., Ltd. (Shanghai, China). The phosphorylated JNK (pJNK, Cat\#07-175) antibody and Triton X-100 (\#T8787) were purchased from Sigma-Aldrich Trading Co., Ltd. (Shanghai, China). The Cy3-conjugated goat anti-rat IgG (\#A0521), 2-(4-Amidinophenyl)-6-indolecarbamidine dihydrochloride (DAPI, \#C1005), phosphate buffered saline (PBS, \#ST476), total superoxide dismutase (T-SOD) assay kit (\#S0101), catalase (CAT) assay kit (\#S0051) and lipid peroxidation (MDA) assay kit (\#S0131) were purchased from Beyotime Biotechnology (Shanghai, China). The 2', $7^{\prime}$-dichlorodihydrofluorescein diacetate $\left(\mathrm{H}_{2}\right.$ DCFDA) was purchased from Thermo Fisher Scientific Co., Ltd. (Shanghai, China). All other reagents used were of analytical grade and commercially available.

\subsection{Fly Husbandry}

The following flies were used: w1118 (V\#60000) was obtained from the Vienna Drosophila RNAi Center (Vienna, Austria). esg-Gal4; UAS-GFP; tub-Gal80 ${ }^{\text {ts }}$ (esg-GAL4 ${ }^{\text {ts }}$ ) and Myo1A-Gal4; UAS-GFP; tub-Gal80 ${ }^{\text {ts }}$ (Myo1A-GAL4 ${ }^{\text {ts }}$ ) flies were kindly gifted from Dr. Lihua Jin (Northeast Forestry University, Harbin, China) [36]. 10 $\times$ STAT-GFP was 
generously provided by Dr. Jose C. Pastor-Pareja (Tsinghua University, Beijing, China) [37]. The esg-GAL4 $4^{\text {ts }}$ and Myo1A-GAL4 ${ }^{\text {ts }}$ flies were collected within $48 \mathrm{~h}$ after eclosing and aged for 5 days before shifting to $2{ }^{\circ} \mathrm{C}$ (restrictive temperature for $\mathrm{Gal} 80^{\mathrm{ts}}$ ) to induce GFP expression. Animals of the other genotypes were cultured under $25{ }^{\circ} \mathrm{C}$ and $60 \%$ humidity with a $12 \mathrm{~h}$ light: $12 \mathrm{~h}$ dark cycle, unless otherwise noted. All flies were cultured on corn-yeast standard food as the previous report [38]. The concentration of UA used in vivo was slightly adjusted according to Staats's research [18].

\subsection{Survival Assays}

For survival experiments, groups of $\sim 3$ days newly emerged flies ( 40 females and 40 males) reared on normal food (NF) or UA-containing food $(100 \mu \mathrm{M})$ were starved for $2 \mathrm{~h}$ before being transferred into vials containing three layers of filter paper soaked with $200 \mu \mathrm{L}$ of $5 \%$ sucrose (control group) or $5 \%$ sucrose solution containing $0.5 \%$ SDS (NF-SDS, UC model group). To summarize, the control group flies were emergenced on normal food for 7 days and then fed with 5\% sucrose (NF-SUC). The UC model group flies were emergenced on normal food for 7 days and then fed with $5 \%$ sucrose supplemented with $0.5 \%$ SDS (NF-SDS). The experimental group flies were emergenced on $100 \mu \mathrm{M}$ UA food for 7 days and then fed with 5\% sucrose supplemented with $0.5 \%$ SDS (UA-SDS). The filter papers and solution were changed once every $24 \mathrm{~h}$. The surviving flies were counted and recorded every day. The experiment was repeated three times.

\subsection{Climbing Ability Assay}

Climbing ability assay was performed as described previously [39]. Ten newly emerged ( 3 day old) flies were maintained on UA-supplemented $(100 \mu \mathrm{M})$ or control diet for 7 days. Then, the flies exposed to sucrose or SDS $(0.5 \%, w / v)$ for $24 \mathrm{~h}$ (as stated in Section 2.3), were collected and placed in plastic cylinder $(1.5 \mathrm{~cm}$ diameter $\times 16 \mathrm{~cm}$ length). The flies were gently hit to ensure all flies down to the bottom of the plastic cylinder. The climbing ability was evaluated by the proportion of flies that climbed upwards more than $10 \mathrm{~cm}$ within $10 \mathrm{~s}$.

\subsection{Intestinal Morphology Analysis}

Twelve newly eclosed ( $\sim 3$ day old) female flies were maintained on UA-supplemented $(100 \mu \mathrm{M})$ or control diet for 7 days and then exposed to sucrose or SDS $(0.5 \%, w / v)$ for $48 \mathrm{~h}$ (as stated in Section 2.3), were dissected in the $1 \times$ PBS and immediately observed under a Nikon ECLIPES Ti2-U microscope (Nikon, Tokyo, Japan)

\subsection{Smurf Assay}

The Smurf assay was performed as previously described with minor modifications [18]. Briefly, newly eclosed female flies were maintained on UA-supplemented $(100 \mu \mathrm{M})$ or control diet for 7 days. Then, the flies were treated with SDS (0.5\% SDS in 5\% sucrose, $w / v)$ or $5 \%$ sucrose as negative control $(w / v)$. For the assay, the flies were starved for $2 \mathrm{~h}$ every other day and then treated for $6 \mathrm{~h}$ with the SDS/sucrose solution soaked on a Whatman filter paper before they were relocated to Brilliant Blue FCF-dyed food. After feeding for 7 days, the flies were frozen to death, photographed and then homogenized with $1 \times$ PBS and centrifuged. The intestine integrity was determined by the absorbance of the supernatant at $625 \mathrm{~nm}$ as the previous reports $[18,40]$.

\subsection{Reactive Oxygen Species Assay}

Adult females were exposed to SDS $(0.5 \%, w / v)$ and incubated at $25^{\circ} \mathrm{C}$ for $48 \mathrm{~h}$. A total of 10 15 intestines were dissected in cold $1 \times$ PBS and incubated in $\mathrm{H}_{2}$ DCFDA ( $5 \mu \mathrm{M}$ in PBS) for $10 \mathrm{~min}$, then washed 3 times in cold $1 \times$ PBS for $5 \mathrm{~min}$, and immediately observed under a Nikon ECLIPES Ti2-U microscope (Nikon, Tokyo, Japan). The data presented are from three independent experiments. 


\subsection{Immunohistochemistry}

For $\mathrm{pJNK}$ staining in intestines, the intestines of female flies were dissected, fixed, stained, and mounted following standard procedures [41,42]. The following antibodies were used: rabbit anti-pJNK (1:200) and Cy3-conjugated goat anti-rat IgG (1:500). For STAT testing in intestines, the intestines of $10 \times$ STAT-GFP flies were dissected and immediately fixed in $4 \%$ paraformaldehyde and washed three times with $0.3 \%$ PBST (PBS containing $0.3 \%$ Triton X-100). For nucleic acid staining, samples were incubated in $50 \mathrm{ng} / \mathrm{mL}$ DAPI for $8 \mathrm{~min}$. Slices were mounted with 50\% glycerol/PBS. Confocal images were taken with a Zeiss LSM710 Meta confocal microscope. ImageJ was employed to quantify the pJNK and STAT-GFP level.

\subsection{Fluorescence Microscopy}

Intestines of esg-GAL4 $4^{\text {ts }}$ and Myo1A-GAL4 $4^{\text {ts }}$ flies were dissected and immediately fixed in $4 \%$ paraformaldehyde, stained, and mounted. Slices were mounted with $50 \%$ glycerol/PBS. Quantification of GFP intensity in the intestine was constructed by measuring $\mathrm{GFP}^{+}$cell fluorescence intensity within posterior midgut [36]. Fluorescence of $\mathrm{GFP}^{+}$cells was measured using ImageJ.

\subsection{Statistical Analysis}

Data were analyzed by using Student's t-test to compare between groups and one-way analysis of variance (ANOVA) for multiple groups. The statistical results were presented as mean \pm SEM. Asterisks indicate the critical levels of significance $\left({ }^{*} p<0.05,{ }^{* *} p<0.01\right.$, and $\left.{ }^{* * *} p<0.001\right)$.

\section{Results}

\subsection{The Intestinal Disruption Induced by SDS in Drosophila Could Be Rescued by UA}

To investigate the effect of UA on chemical-induced UC, we produced a Drosophila UC model induced by the inflammatory reagent SDS (NF-SDS). The UC model group flies (NF-SDS) showed shorter life spans and decreased mobility compared with the control group (NF-SUC) (Figure 1A-D). As shown in Figure 1A,B, flies which were pre-fed with $100 \mu \mathrm{M}$ UA (UA-SDS) showed a significant resistance to SDS. Following treatment with SDS for $108 \mathrm{~h}$, the survival rates of female and male flies were dramatically increased from $0 \%$ to $45 \%(p<0.001)$ and from $2.5 \%$ to $30 \%(p<0.001)$ in the UA-SDS group, respectively, compared with the NF-SDS group (Figure 1A,B). In addition, SDS exposure induced significant reduction of climbing ability (female down 54\%; male down 38\%); the decreased climbing abilities were weaker in UA-SDS flies (female, showing a 35\% increase; male, showing a $24 \%$ increase) (Figure 1C,D). These results indicated that UA could increase the survival rate and climbing activity of flies treated with toxic chemical SDS. Taken together, UA showed protection against chemical-induced UC in Drosophila.

\subsection{Protective Effect of UA against Morphological Changes in the Drosophila Intestine following Treatment with SDS}

The male flies and female flies upon SDS exposure exhibited largely similar phenotypes, except that the phenotypes of the female flies were more severe than those of the male flies. In addition, the protection of UA on female flies against SDS was better than the males. Therefore, only the results of female flies are shown hereafter. As shown in Figure 2A,B, the intestines of UC model flies (NF-SDS) appeared shorter by $57 \%$ than the control (NF-SUC). These results were consistent with previous studies [34,35]. Compared with the UC model group, the intestine length of flies in the UA-SDS group was extended by $64 \%(p<0.001)$. In addition, the intestinal epithelium is susceptible to damage caused by the chemical compound SDS [35]. SDS-induced colitis showed disrupted intestinal barrier integrity $[34,43]$. The intestinal barrier function could be evaluated by using a blue dye, Brilliant Blue FCF. Loss of intestinal barrier function showed the Smurf phenotype with blue dye throughout the whole body of flies, which could be quantified by Smurf- 
ness $[44,45]$. To investigate the protective effect of UA on UC, the Smurf assay was used to evaluate intestine integrity of flies. After exposure to SDS, the flies showed enhanced blue distribution when compared to the control, showing a 304\% increase in Smurfness (Figure 2C,D). This suggests that exposure to SDS increased intestinal permeability, indicating damage to the epithelial barrier. UA supplementation alleviated the epithelial damage, even to the normal level (Figure 2C,D). Taken together, our findings suggested that UA protects against disruption of the intestinal barrier caused by SDS in Drosophila.

A

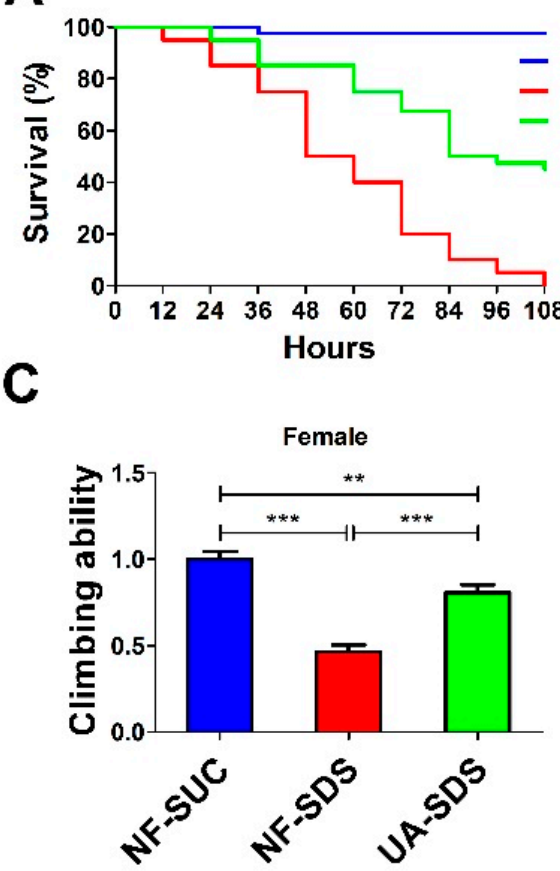

B

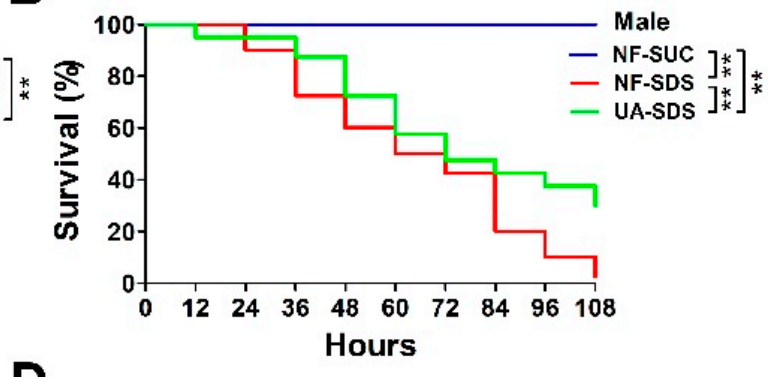

D

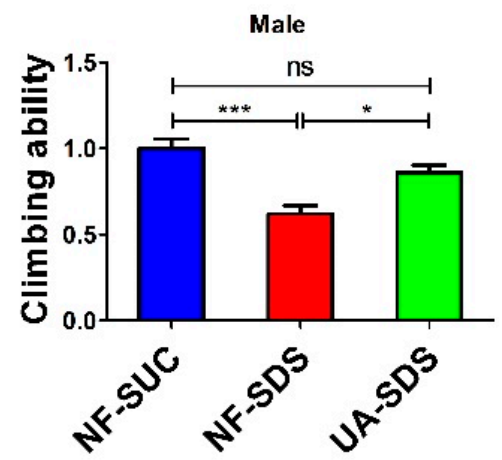

Figure 1. The reduced survival rate and climbing ability caused by sodium dodecyl sulfate (SDS) could be improved by ulcerative colitis (UA) in Drosophila. (A,B) The ulcerative colitis (UC) model group (NF-SDS) showed shorter life span than control group (NF-SUC). The $100 \mu \mathrm{M}$ UA supplementation (UA-SDS) can significantly elevate the decreased survival rates of both female (A) and male (B) flies exposed to $0.5 \%$ SDS (NF-SDS). (C,D) The UC model group (NF-SDS) showed decreased climbing ability compared to the control group (NF-SUC). UA supplementation (UA-SDS) elevated the reduced climbing ability of female (C) and male (D) flies treated with $0.5 \%$ SDS. ${ }^{*} p<0.05$, ** $p<0.01,{ }^{* * *} p<0.001$, ns: not significant.

\subsection{UA Protected Intestinal Stem Cells from SDS-Induced Proliferation and Differentiation}

Intestinal damage caused by chemicals results in the activation of ISC proliferation to regenerate the damaged intestinal epithelium [46]. To evaluate the protective effect of UA on the intestinal homeostasis, SDS was employed to stimulate the proliferation and differentiation of ISCs [16]. The numbers of ISCs/enteroblasts (EBs) were detected by GFP expression driven by esg-Gal4 ${ }^{\text {ts }}$ (as revealed by fluorescence intensity of $\mathrm{GFP}^{+}$ cells) [47]. After $48 \mathrm{~h}$ of exposure to SDS, the $\mathrm{GFP}^{+}$cells were clustered and showed aberrant morphology (Figure 3A). A statistically dramatic increase in ISCs/EBs was observed in the NF-SDS group, showing a 4-fold increase in fluorescence intensity when compared to the control group (Figure 3B). UA supplementation clearly restored the aberrated morphology of ISCs/EBs and showed a strong reduction in the number of ISC and EB cells (down 44\%) (Figure 3A,B). 


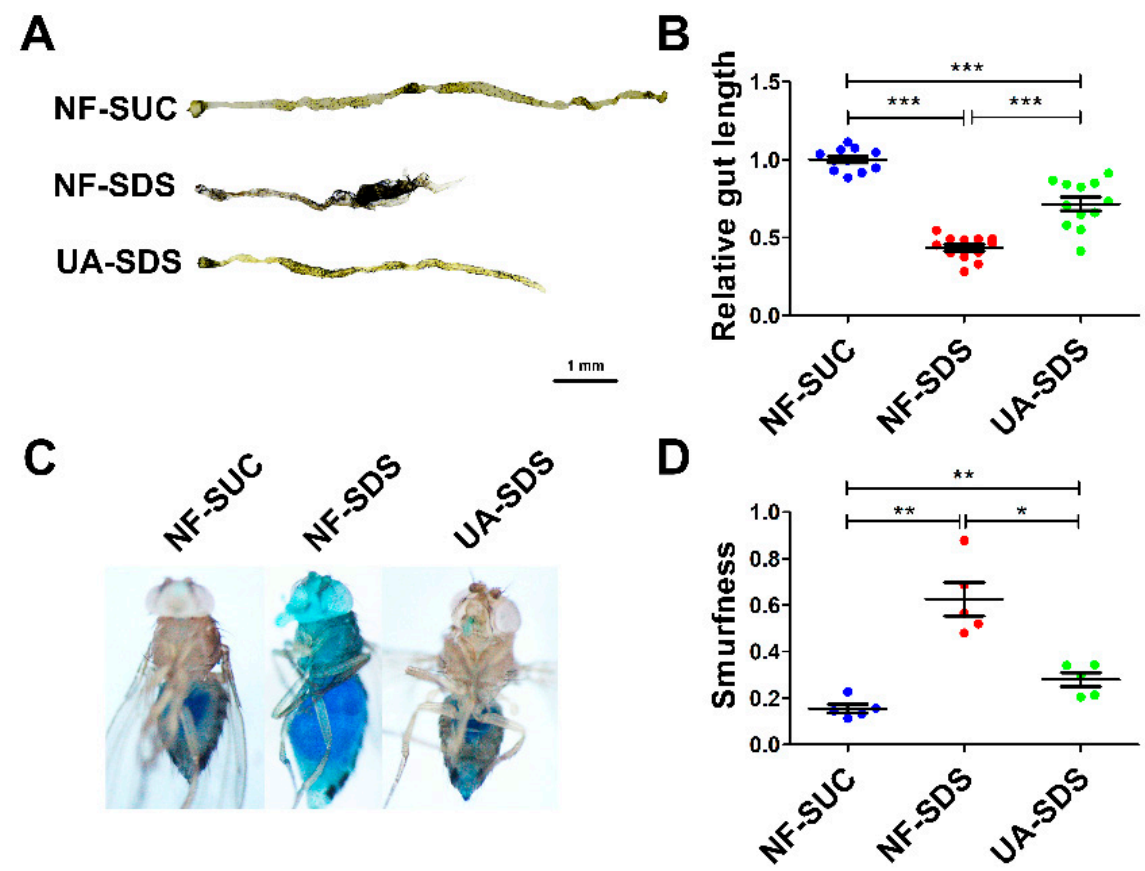

Figure 2. UA alleviated SDS-induced impairment of intestinal morphology and barrier integrity. (A) After exposure to $0.5 \% \mathrm{SDS}$ for $48 \mathrm{~h}$, the intestines of these flies (NF-SDS) appeared shorter than control (NF-SUC), UA significantly increased the intestine length. (B) Quantitative measurement of (A) ( $\mathrm{n} \geq 10$ ). (C) A small amount of blue dye occurred in the gut of control flies (NF-SUC) while the blue dye escaped throughout the whole body of UC model flies exposed to $0.5 \%$ SDS for $48 \mathrm{~h}$ (NFSDS), which can be prevented by pre-feeding of UA (UA-SDS). (D) The intestinal barrier permeability was quantified by measuring the Smurfness $(n=5){ }^{*} p<0.05,{ }^{* *} p<0.01,{ }^{* * *} p<0.001$.

A

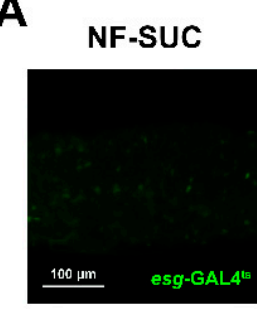

C

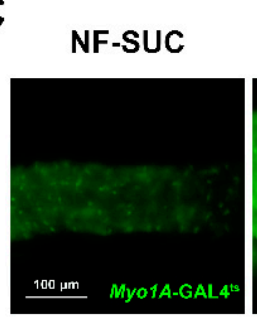

NF-SDS

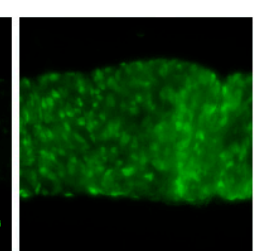

NF-SDS

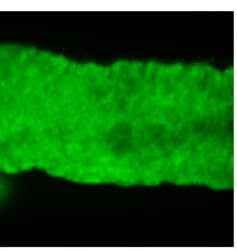

UA-SDS

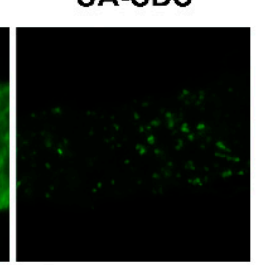

D
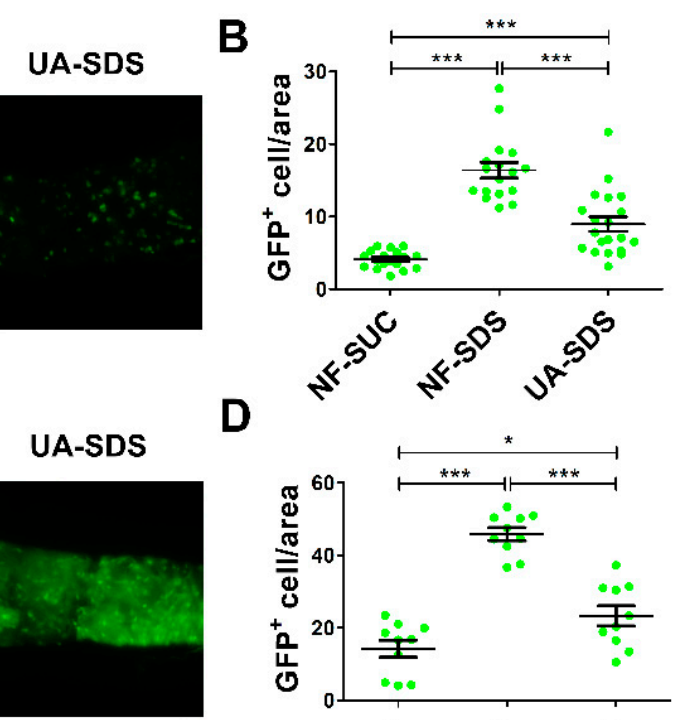

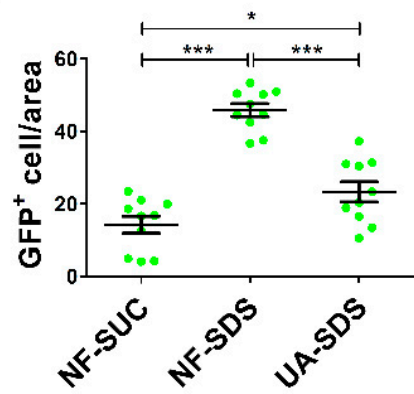

Figure 3. UA protected intestine from SDS-induced intestinal stem cell (ISC) proliferation and differentiation. (A) UA could significantly reduce proliferation and differentiation of ISCs/ enteroblasts (EBs). (B) Fluorescence intensity of the $\mathrm{GFP}^{+}$ISCs/EBs in panels ( $\mathrm{n} \geq 15$ ). (C) UA could strongly decrease SDS-induced EC proliferation. (D) Fluorescence intensity of the GFP ${ }^{+}$EC cells in panels $(\mathrm{n} \geq 10) .{ }^{*} p<0.05,{ }^{* * *} p<0.001$. 
Myo1A is specifically expressed in enterocytes (ECs) [48]. Subsequently, we used the GFP level of Myo1A-GAL4 ${ }^{\text {ts }}$ to analyze the numbers of ECs. As shown in Figure 3C,D, we found that SDS could promote EBs to further differentiate into ECs in the NF-SDS group (a 2.2-fold increase in fluorescence intensity of $M y 01 A \mathrm{GFP}^{+}$cells, compared with the control group). As expected, the increased ECs in the NF-SDS group could be significantly attenuated by UA; however, the number of ECs in the UA-SDS group remained larger than the control group $(p<0.05)$. These results indicated that the ISCs proliferation and differentiation induced by SDS could be rescued by UA.

\subsection{UA Protects Intestine against SDS-Induced Oxidative Damage}

Environmental stresses cause excessive ROS, which induces cellular oxidative damage [49,50], ISC activation and tissue renewal [51]. Recent studies revealed that UA could significantly alleviate the ROS production and cell death induced by a mycotoxin ochratoxin A [52]. Then, we tested whether UA could attenuate the excessive ROS levels in the damaged intestine. $\mathrm{H}_{2}$ DCFDA, a cell-permeable probe, was used to detect the intracellular ROS levels. Adult flies were exposed to SDS for $48 \mathrm{~h}$ and a robust fluorescence signal was observed in NF-SDS compared with control. The enhanced fluorescence signal in the posterior midgut of the NF-SDS flies was markedly reduced in the UA-SDS group flies (Figure 4A). Statistical analysis indicated that UA showed a significant attenuation in the fluorescence signal (down 57\%, $p<0.05$ ) compared with the UC model group (Figure 4B). Additionally, SDS exposure caused a significant increase in MDA, a marker of lipid peroxidative damage [53], showing a 167\% increase compared with control. Treatment with UA significantly reversed the SDS-induced MDA accumulation (Figure 4C). These findings suggested that UA protected the adult flies against SDS-induced oxidative damage.

Many antioxidant enzymes, such as superoxide dismutase (SOD) and catalase (CAT), help organisms resist oxidative damage [54]. To further investigate how UA protects against SDS-induced ROS, the activities of these antioxidant enzymes were examined. As shown in Figure 4D,E, the activity of either total SOD (T-SOD) or CAT was strongly downregulated upon SDS exposure (decreased by $52 \%$ and $45 \%$, respectively, compared to the control), whereas UA supplementation restored the activities of these enzymes (30\% and $27 \%$ increase in T-SOD and CAT activity respectively, compared to NF-SDS group). Collectively, the results suggested that UA may maintain the host redox homeostasis following SDS exposure, therefore protecting against chemical-induced ISC proliferation and differentiation.

\subsection{UA Protects against SDS-Induced Intestine Damage by Regulating JNK Signaling Pathway}

Many studies demonstrated that ROS can activate the JNK signaling pathway, and the ROS/JNK signaling plays a vital role in environmental stress responses [55]. Moreover, the ROS-induced JNK signaling can boost ISC proliferation [56]. To further assess whether the JNK signaling participates in the protection of UA on the intestine, the pJNK levels were detected as an indicator of JNK signaling [57]. After exposure to SDS for $48 \mathrm{~h}$, the posterior intestine of UC model flies (NF-SDS) suffered severe injury, and the expression of pJNK was dramatically increased (a 2.27-fold increase, compared with control) (Figure 5A,B). In addition, we found that SDS treatment led to aberrant nucleus morphology, clustered nuclei and enlarged size (increase of 55\%) (Figure S1), which mimics the results in DSS treatment [36]. Compared with the NF-SDS group, UA supplementation showed a significant attenuation in pJNK level (down 40\%) and rescued the enlarged nuclear size (Figure 5A,B and Figure S1). Collectively, the results demonstrated that UA may protect the Drosophila intestine from SDS-induced damage through the JNK signaling pathway. 


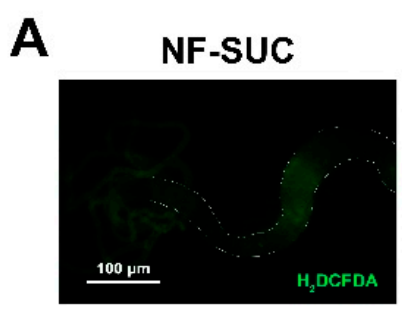

B

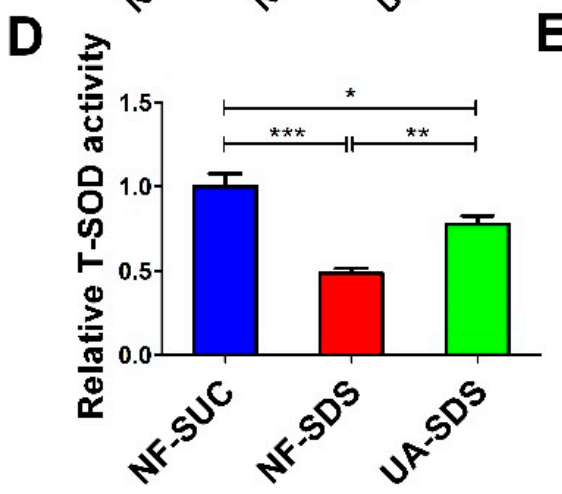

C
NF-SDS

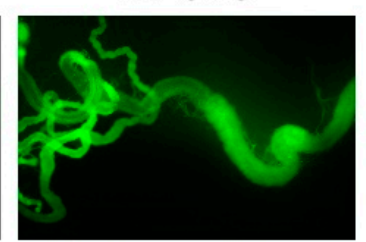

UA-SDS
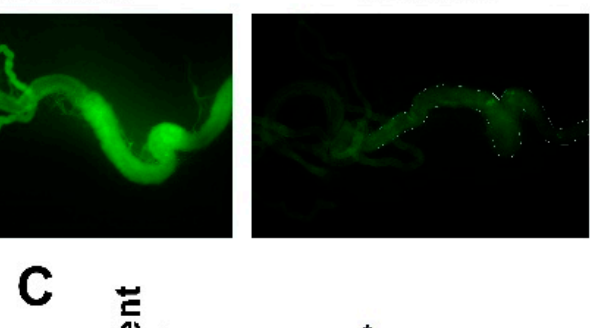

E
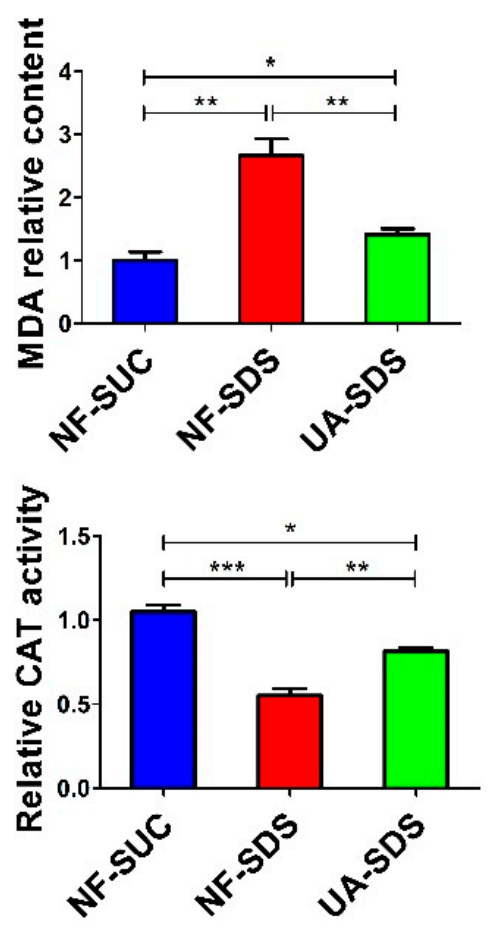

Figure 4. UA protected intestine from SDS-induced oxidative damage. (A) UA protected epithelial cells against SDS-induced oxidative stress. $\mathrm{H}_{2}$ DCFDA staining of the anterior midgut of a female fly after ingestion of $0.5 \%$ for $48 \mathrm{~h}$. (B) Fluorescence intensity of ROS levels in panels ( $\mathrm{n} \geq 10$ ). (C-E) UA supplementation alleviated malondialdehyde (MDA) levels and increased the activities of total superoxide dismutase (T-SOD) and catalase (CAT), after exposure to SDS for $48 \mathrm{~h} .{ }^{*} p<0.05$, ** $p<0.01, * * * p<0.001$.

A
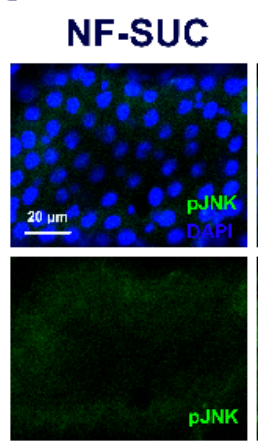
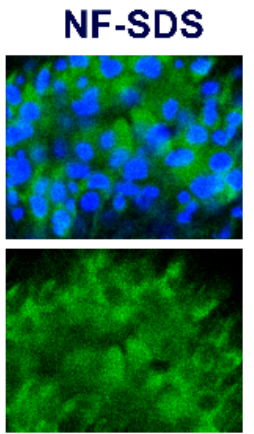
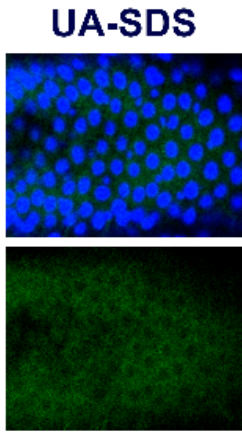

B

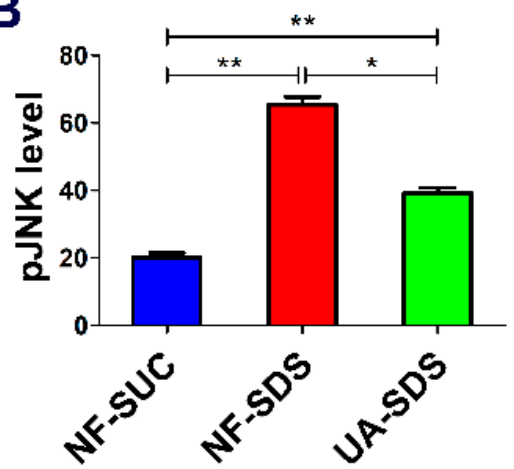

Figure 5. UA alleviated SDS-induced intestine damage by inhibiting c-Jun N-terminal kinase (JNK) signaling. (A) Immunohistochemistry indicated that the induced level of phosphorylated JNK (pJNK) as well as the aberrant nucleus morphology caused by SDS were attenuated by UA. (B) Quantification of pJNK levels in the intestines $(\mathrm{n} \geq 6){ }^{*} p<0.05,{ }^{* *} p<0.01$. 


\subsection{JAK/STAT Pathway Is Involved in the Protection of UA on Drosophila Intestine Homeostasis}

JNK acts synergistically with JAK/STAT in response to tissue damage in fly tissues [58]. Intestine damage caused by feeding flies with chemicals stimulates ISC proliferation through the JNK-regulated JAK/STAT pathway [59]. Given that chemical SDS is long known to cause JAK/STAT activation [44], we examined whether the JAK/STAT signaling participates in UA's protective mechanism. As shown in Figure 6A,B, the STAT activity was obviously activated in the NF-SDS group (feeding SDS for $48 \mathrm{~h}$ ) as monitored by expression of $10 \times$ STAT-GFP (a 17.7-fold increase), compared with the control group. Pre-supplemented UA could alleviate the upregulated expression of $10 \times$ STATGFP [37] (down 69\%) (Figure 6A,B). These data suggested that UA may regulate the JNK/JAK/STAT responsive module to maintain Drosophila intestine homeostasis from toxic chemical-induced disruption.

A
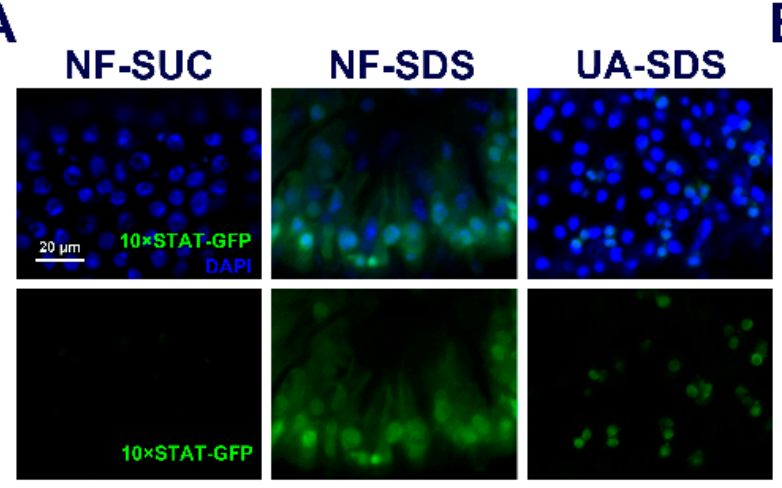

B

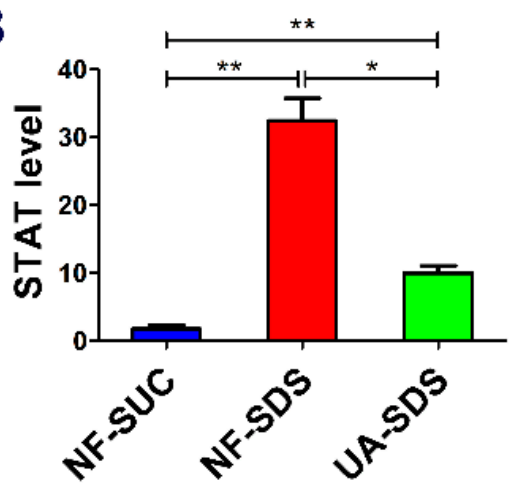

Figure 6. UA protected against SDS-induced injury via regulating JAK/STAT signaling pathway. (A) The JAK/STAT signaling was activated by SDS, and UA significantly attenuated the SDS-induced JAK/STAT activation. (B) Quantification of $10 \times$ STAT-GFP levels in the intestines $(\mathrm{n} \geq 6) .{ }^{*} p<0.05$, ** $p<0.01$.

\section{Discussion}

The intestinal epithelium is a prominent defense barrier against environmental stresses [60], and the disruption of intestinal integrity is one of the primary causes of several intestinal disorders. Herein, we investigated the function of UA in protecting against SDS-induced intestine damage. The results demonstrated that pre-supplemented UA significantly increased the survival rates and locomotor activity of flies cultured on SDS. Moreover, UA could restore the ISC proliferation and differentiation induced by SDS. Further study indicated that the protection of UA on Drosophila intestine homeostasis is mediated by the reduction of the JNK/JAK/STAT signaling pathway. These results provide in vivo evidence for the protective effect of UA on SDS-induced intestinal dysfunction. Therefore, our findings may provide a theoretical basis for functional food or natural medicine development of UA.

ROS plays an important role in maintaining intestinal homeostasis and improving stress tolerance in organisms [49]. Excessive ROS have been implicated in toxic chemicalinduced intestinal disorders [61]. Enterocytes sense ROS and then regulate the proliferation and differentiation of ISCs and therefore maintain intestinal health and homeostasis [16,62]. Here we found that SDS significantly increased the ROS-induced ISC cell proliferation and differentiation (Figure 4A,B), which was consistent with Zhang's research that DSS induced the ISC cell proliferation and differentiation [36]. It has been reported that UA could reduce ROS generation in mesangial cells and alleviated mesangial cell injury [19]. In addition, UA elevated the activities of T-SOD and CAT responsible for scavenging ROS (Figure 4D,E). Therefore, we concluded that UA may increase the activities of several ROS-scavenging antioxidant enzymes and regulate the SDS-induced ISC proliferation and differentiation.

Several signaling pathways have been implicated in maintaining intestinal homeostasis, such as JNK, JAK/STAT, Notch and Hippo pathways [63-65]. Furthermore, a 
coordinated activation of conserved signaling modules, such as JNK and JAK/STAT, plays crucial roles in tissue restoration in response to environmental stress and injury [66]. For example, the crosstalk between JNK and Hippo signaling regulates ISC proliferation response to stress $[63,67]$. Previous studies have shown that the activation of JNK signaling caused by environmental stresses in ECs can induce cytokine upd3 expression, which triggers the JAK/STAT pathway required for ISC proliferation in intestinal regeneration $[44,68]$. In this study, we found that UA could inhibit the JNK signaling by attenuating the phosphorylation of JNK and then reducing the SDS-induced JAK/STAT activation (Figure 6A,B). Therefore, we proposed that UA may protect UC by restoring the ISC proliferation and ROS production by inhibiting the JNK/JAK/STAT pathway and enhancing the resistance of Drosophila to environmental challenges. It is unknown whether there are other pathways that participate in this process. Additional protective molecular pathways stimulated as response to UA may be further explored by using high-throughput transcriptome-wide RNA sequencing in the future. Even though Drosophila has many organs that function like mammalian organs [32], there are some morphological differences. It is reported that approximately $60 \%$ of the Drosophila genome is homologous to that of humans [26], and whether the other $40 \%$ genome is involved in this process remains unknown. Therefore, it would be significant if our findings could be verified in mammals or human cell lines in the future. Taken together, our results indicate that a high intake of fruits and vegetables rich in UA, such as apple, cranberry, daylily and so on, may protect against intestinal injury induced by environmental stresses.

\section{Conclusions}

In summary, UA could protect Drosophila adults from SDS-induced intestinal damage. The behavioral defects, morphological defects and the ROS production caused by SDS could be rescued by UA. Further studies indicated that UA could remarkably attenuate the SDS-induced excessive ISC proliferation and differentiation. Moreover, the protective effect of UA on UC is mediated by the JNK/JAK/STAT signaling pathway. Therefore, our data provided evidence for UA to be potentially developed as promising alternative functional foods and medicines for UC.

Supplementary Materials: The following are available online at https:/ /www.mdpi.com/article/10 .3390 /antiox11020426/s1, Figure S1: UA significantly restored SDS-induced nuclear enlargement.

Author Contributions: Conceptualization, G.X.; Data curation, T.W., L.W., X.J. and Y.G.; Formal analysis, T.W. and G.X.; Funding acquisition, G.X.; Investigation, T.W. and G.X.; Methodology, T.W.; Project administration, G.X.; Supervision, G.X.; Validation, T.W., L.W., X.J. and Y.G.; Writing-original draft, T.W.; Writing-review and editing, G.X. All authors have read and agreed to the published version of the manuscript.

Funding: This research was funded by the National Natural Science Foundation of China (31671284 and 32170576), Fundamental Research Funds for the Central Universities (JZ2020HGPA0115), and Youth Science and Technology Talents Support Program (2020) by Anhui Association for Science and Technology (RCTJ202001).

Institutional Review Board Statement: Not applicable.

Informed Consent Statement: Not applicable.

Data Availability Statement: The data presented in this study are available in the article and supplementary materials.

Acknowledgments: The authors appreciate the gifts of fly stocks from Jose C. Pastor-Pareja (Tsinghua University, Beijing, China) and Lihua Jin (Northeast Forestry University, Harbin, China). The authors are also grateful to the Bloomington Drosophila Stock Center and the Vienna Drosophila RNAi Center.

Conflicts of Interest: The authors declare no conflict of interest. The funders had no role in the design of the study; in the collection, analyses, or interpretation of data; in the writing of the manuscript, or in the decision to publish the results. 


\section{References}

1. Cosnes, J.; Gower-Rousseau, C.; Seksik, P.; Cortot, A. Epidemiology and natural history of inflammatory bowel diseases. Gastroenterology 2011, 140, 1785-1794. [CrossRef] [PubMed]

2. Long, M.; Shou, J.; Wang, J.; Hu, W.; Hannan, F.; Mwamba, T.M.; Farooq, M.A.; Zhou, W.; Islam, F. Ursolic acid limits salt-induced oxidative damage by interfering with nitric oxide production and oxidative defense machinery in rice. Front. Plant Sci. 2020, 11, 697. [CrossRef] [PubMed]

3. Singh, S.; Facciorusso, A.; Dulai, P.S.; Jairath, V.; Sandborn, W.J. Comparative risk of serious infections with biologic and/or immunosuppressive therapy in patients with inflammatory bowel diseases: A systematic review and meta-analysis. Clin. Gastroenterol. Hepatol. 2020, 18, 69-81.e3. [CrossRef] [PubMed]

4. Akram, W.; Garud, N.; Joshi, R. Role of inulin as prebiotics on inflammatory bowel disease. Drug Discov. Ther. 2019, 13, 1-8 [CrossRef]

5. Ley, R.E.; Peterson, D.A.; Gordon, J.I. Ecological and evolutionary forces shaping microbial diversity in the human intestine. Cell 2006, 124, 837-848. [CrossRef]

6. Radtke, F.; Clevers, H. Self-renewal and cancer of the gut: Two sides of a coin. Science 2005, 307, 1904-1909. [CrossRef]

7. Raphael, T.J.; Kuttan, G. Effect of naturally occurring triterpenoids glycyrrhizic acid, ursolic acid, oleanolic acid and nomilin on the immune system. Phytomedicine 2003, 10, 483-489. [CrossRef]

8. Ikeda, Y.; Murakami, A.; Ohigashi, H. Ursolic acid: An anti- and pro-inflammatory, triterpenoid. Mol. Nutr. Food Res. 2008, 52, 26-42. [CrossRef]

9. Checker, R.; Sandur, S.K.; Sharma, D.; Patwardhan, R.S.; Jayakumar, S.; Kohli, V.; Sethi, G.; Aggarwal, B.B.; Sainis, K.B. Potent anti-inflammatory activity of ursolic acid, a triterpenoid antioxidant, is mediated through suppression of NF-kappaB, AP-1 and NF-AT. PLoS ONE 2012, 7, e31318. [CrossRef]

10. Ali, M.S.; Ibrahim, S.A.; Jalil, S.; Choudhary, M.I. Ursolic acid: A potent inhibitor of superoxides produced in the cellular system Phytother. Res. 2007, 21, 558-561. [CrossRef]

11. Yeh, C.T.; Wu, C.H.; Yen, G.C. Ursolic acid, a naturally occurring triterpenoid, suppresses migration and invasion of human breast cancer cells by modulating c-Jun N-terminal kinase, Akt and mammalian target of rapamycin signaling. Mol. Nutr. Food Res. 2010, 54, 1285-1295. [CrossRef] [PubMed]

12. Ku, C.M.; Lin, J.Y. Anti-inflammatory effects of 27 selected terpenoid compounds tested through modulating Th1/Th2 cytokine secretion profiles using murine primary splenocytes. Food Chem. 2013, 141, 1104-1113. [CrossRef] [PubMed]

13. Do Nascimento, P.G.G.; Lemos, T.L.G.; Bizerra, A.M.C.; Arriaga, A.M.C.; Ferreira, D.A.; Santiago, G.M.P.; Braz Filho, R.; Costa, J.G.M. Antibacterial and antioxidant activities of ursolic acid and derivatives. Molecules 2014, 19, 1317-1327. [CrossRef] [PubMed]

14. Shi, Y.; Leng, Y.; Liu, D.; Liu, X.; Ren, Y.; Zhang, J.; Chen, F. Research advances in protective effects of ursolic acid and oleanolic acid against gastrointestinal diseases. Am. J. Chin. Med. 2021, 49, 413-435. [CrossRef] [PubMed]

15. Wan, S.Z.; Liu, C.; Huang, C.K.; Luo, F.Y.; Zhu, X. Ursolic acid improves intestinal damage and bacterial dysbiosis in liver fibrosis mice. Front. Pharmacol. 2019, 10, 1321. [CrossRef]

16. Amcheslavsky, A.; Jiang, J.; Ip, Y.T. Tissue damage-induced intestinal stem cell division in Drosophila. Cell Stem Cell 2009, 4, 49-61. [CrossRef]

17. Li, J.; Liang, X.; Yang, X. Ursolic acid inhibits growth and induces apoptosis in gemcitabine-resistant human pancreatic cancer via the JNK and PI3K/Akt/NF-kappaB pathways. Oncol. Rep. 2012, 28, 501-510. [CrossRef]

18. Staats, S.; Wagner, A.E.; Luersen, K.; Kunstner, A.; Meyer, T.; Kahns, A.K.; Derer, S.; Graspeuntner, S.; Rupp, J.; Busch, H.; et al Dietary ursolic acid improves health span and life span in male Drosophila melanogaster. Biofactors 2019, 45, 169-186. [CrossRef]

19. Wang, E.M.; Fan, Q.L.; Yue, Y.; Xu, L. Ursolic acid attenuates high glucose-mediated mesangial cell injury by inhibiting the phosphatidylinositol 3-kinase/Akt/mammalian target of rapamycin (PI3K/Akt/mTOR) signaling pathway. Med. Sci. Monit. 2018, 24, 846-854. [CrossRef]

20. Liu, B.H.; Piao, X.H.; Guo, L.Y.; Liu, S.S.; Chai, F.; Gao, L.M. Ursolic acid protects against ulcerative colitis via anti-inflammatory and antioxidant effects in mice. Mol. Med. Rep. 2016, 13, 4779-4785. [CrossRef]

21. Redza-Dutordoir, M.; Averill-Bates, D.A. Activation of apoptosis signalling pathways by reactive oxygen species. Biochim. Biophys. Acta Mol. Cell Res. 2016, 1863, 2977-2992. [CrossRef] [PubMed]

22. Chao, L.; Zheng, P.Y.; Xia, L.; Yong, Y.; Lu, G.F.; Tang, F.; Zhao, Z.G. Calycosin attenuates dextran sulfate sodium (DSS)-induced experimental colitis. Iran J. Basic Med. Sci. 2017, 20, 1056-1062. [PubMed]

23. Redhai, S.; Boutros, M. The role of organelles in intestinal function, physiology, and disease. Trends Cell Biol. 2021, 31, 485-499. [CrossRef] [PubMed]

24. Wang, C.; Guo, X.; Xi, R. EGFR and Notch signaling respectively regulate proliferative activity and multiple cell lineage differentiation of Drosophila gastric stem cells. Cell Res. 2014, 24, 610-627. [CrossRef] [PubMed]

25. Liu, T.S.; Ma, H.Y.; Shi, W.; Duan, J.L.; Wang, Y.; Zhang, C.T.; Li, C.L.; Lin, J.; Li, S.; Lv, J.G.; et al. Inhibition of STAT3 signaling pathway by ursolic acid suppresses growth of hepatocellular carcinoma. Int. J. Oncol. 2017, 51, 555-562. [CrossRef]

26. Ugur, B.; Chen, K.; Bellen, H.J. Drosophila tools and assays for the study of human diseases. Dis. Model. Mech. 2016, 9, 235-244. [CrossRef]

27. Xiao, G.; Zhou, B. What can flies tell us about zinc homeostasis? Arch. Biochem. Biophys. 2016, 611, 134-141. [CrossRef] 
28. Guo, X.; Lv, J.; Xi, R. The specification and function of enteroendocrine cells in Drosophila and mammals: A comparative review. FEBS J. 2021. Epub ahead of print. [CrossRef]

29. Hung, R.J.; Li, J.S.S.; Liu, Y.; Perrimon, N. Defining cell types and lineage in the Drosophila midgut using single cell transcriptomics Curr. Opin. Insect Sci. 2021, 47, 12-17. [CrossRef]

30. Boumard, B.; Bardin, A.J. An amuse-bouche of stem cell regulation: Underlying principles and mechanisms from adult Drosophila intestinal stem cells. Curr. Opin. Cell Biol. 2021, 73, 58-68. [CrossRef]

31. Reiter, L.T.; Potocki, L.; Chien, S.; Gribskov, M.; Bier, E. A systematic analysis of human disease-associated gene sequences in Drosophila melanogaster. Genome Res. 2001, 11, 1114-1125. [CrossRef] [PubMed]

32. Apidianakis, Y.; Rahme, L.G. Drosophila melanogaster as a model for human intestinal infection and pathology. Dis. Model Mech. 2011, 4, 21-30. [CrossRef] [PubMed]

33. Suzuki, R.; Gunarta, I.K.; Boldbaatar, J.; Erdenebaatar, P.; Odongoo, R.; Yoshioka, K. Functional role of c-Jun NH2-terminal kinase-associated leucine zipper protein (JLP) in lysosome localization and autophagy. Drug Discov. Ther. 2020, 14, 35-41. [CrossRef] [PubMed]

34. Zhou, Y.; Liu, Z.; Chen, Y.; Jin, L.H. Identification of the protective effects of traditional medicinal plants against SDS-induced Drosophila gut damage. Exp. Ther. Med. 2016, 12, 2671-2680. [CrossRef]

35. Liu, Z.; Chen, Y.; Zhang, H.; Jin, L.H. Crocus sativus L. protects against SDS-induced intestinal damage and extends lifespan in Drosophila melanogaster. Mol. Med. Rep. 2016, 14, 5601-5606. [CrossRef]

36. Zhang, H.; Wang, S.H.; Jin, L. Acanthopanax senticosus polysaccharide regulates the intestinal homeostasis disruption induced by toxic chemicals in Drosophila. Phytother. Res. 2020, 34, 193-200. [CrossRef]

37. Zang, Y.R.; Wan, M.; Liu, M.; Ke, H.M.; Ma, S.C.; Liu, L.P.; Ni, J.Q.; Pastor-Pareja, J.C. Plasma membrane overgrowth causes fibrotic collagen accumulation and immune activation in Drosophila adipocytes. eLife 2015, 4, e07187. [CrossRef]

38. Wei, T.; Ji, X.W.; Xue, J.S.; Gao, Y.; Zhu, X.M.; Xiao, G.R. Cyanidin-3-O-glucoside represses tumor growth and invasion in vivo by suppressing autophagy via inhibition of the JNK signaling pathways. Food Funct. 2021, 12, 387-396. [CrossRef]

39. Xiao, G.; Fan, Q.; Wang, X.; Zhou, B. Huntington disease arises from a combinatory toxicity of polyglutamine and copper binding Proc. Natl. Acad. Sci. USA 2013, 110, 14995-15000. [CrossRef]

40. Chen, S.Y.; Yang, Q.; Chen, X.; Tian, Y.Q.; Liu, Z.Y.; Wang, S.Y. Bioactive peptides derived from crimson snapper and in vivo anti-aging effects on fat diet-induced high fat Drosophila melanogaster. Food Funct. 2020, 11, 524-533. [CrossRef]

41. Xiao, G.R.; Wan, Z.H.; Fan, Q.W.; Tang, X.N.; Zhou, B. The metal transporter ZIP13 supplies iron into the secretory pathway in Drosophila melanogaster. eLife 2014, 3, e03191. [CrossRef] [PubMed]

42. Yang, F.; Hong, J.; Xiao, G.; Feng, Y.; Peng, R.; Wang, M.; Qu, H. Descemet stripping endothelial keratoplasty in pediatric patients with congenital hereditary endothelial dystrophy. Am. J. Ophthalmol. 2019, 209, 132-140. [CrossRef] [PubMed]

43. Kwon, J.; Lee, C.; Heo, S.; Kim, B.; Hyun, C.K. DSS-induced colitis is associated with adipose tissue dysfunction and disrupted hepatic lipid metabolism leading to hepatosteatosis and dyslipidemia in mice. Sci. Rep. 2021, 11, 5283. [CrossRef] [PubMed]

44. Patel, P.H.; Penalva, C.; Kardorff, M.; Roca, M.; Pavlovic, B.; Thiel, A.; Teleman, A.A.; Edgar, B.A. Damage sensing by a Nox-Ask1-MKK3-p38 signaling pathway mediates regeneration in the adult Drosophila midgut. Nat. Commun. 2019, 10, 4365. [CrossRef]

45. Dambroise, E.; Monnier, L.; Ruisheng, L.; Aguilaniu, H.; Joly, J.S.; Tricoire, H.; Rera, M. Two phases of aging separated by the Smurf transition as a public path to death. Sci. Rep. 2016, 6, 23523. [CrossRef]

46. Kux, K.; Pitsouli, C. Tissue communication in regenerative inflammatory signaling: Lessons from the fly gut. Front. Cell Infect. Microbiol. 2014, 4, 49. [CrossRef]

47. Loza-Coll, M.A.; Southall, T.D.; Sandall, S.L.; Brand, A.H.; Jones, D.L. Regulation of Drosophila intestinal stem cell maintenance and differentiation by the transcription factor Escargot. EMBO J. 2014, 33, 2983-2996. [CrossRef]

48. Zhai, Z.; Boquete, J.P.; Lemaitre, B. Cell-specific imd-nf-kappab responses enable simultaneous antibacterial immunity and intestinal epithelial cell shedding upon bacterial infection. Immunity 2018, 48, 897-910.e7. [CrossRef]

49. Bhattacharyya, A.; Chattopadhyay, R.; Mitra, S.; Crowe, S.E. Oxidative stress: An essential factor in the pathogenesis of gastrointestinal mucosal diseases. Physiol. Rev. 2014, 94, 329-354. [CrossRef]

50. Sun, J.; Fang, R.; Wang, H.; Xu, D.X.; Yang, J.; Huang, X.; Cozzolino, D.; Fang, M.; Huang, Y. A review of environmental metabolism disrupting chemicals and effect biomarkers associating disease risks: Where exposomics meets metabolomics. Environ. Int. 2022, 158, 106941. [CrossRef]

51. Morris, O.; Jasper, H. Reactive oxygen species in intestinal stem cell metabolism, fate and function. Free Radic. Biol. Med. 2021, 166, 140-146. [CrossRef] [PubMed]

52. Li, C.; Chen, W.; Zheng, L.; Zhang, B.; Yang, X.; Zhang, Q.; Wang, N.; Wang, Y.; Yang, J.; Sha, J.; et al. Ameliorative effect of ursolic acid on ochratoxin A-induced renal cytotoxicity mediated by Lonp1/Aco2/Hsp75. Toxicon 2019, 168, 141-146. [CrossRef] [PubMed]

53. Frijhoff, J.; Winyard, P.G.; Zarkovic, N.; Davies, S.S.; Stocker, R.; Cheng, D.; Knight, A.R.; Taylor, E.L.; Oettrich, J.; Ruskovska, T.; et al. Clinical relevance of biomarkers of oxidative stress. Antioxid. Redox Signal. 2015, 23, 1144-1170. [CrossRef] [PubMed]

54. Jeeva, J.S.; Sunitha, J.; Ananthalakshmi, R.; Rajkumari, S.; Ramesh, M.; Krishnan, R. Enzymatic antioxidants and its role in oral diseases. J. Pharm. Bioallied Sci. 2015, 7, S331-S333. [CrossRef] [PubMed] 
55. Shen, H.M.; Liu, Z.G. JNK signaling pathway is a key modulator in cell death mediated by reactive oxygen and nitrogen species. Free Radic. Bio. Med. 2006, 40, 928-939. [CrossRef] [PubMed]

56. Nagai, H.; Kurata, S.; Yano, T. Immunoglobulin superfamily beat-Ib mediates intestinal regeneration induced by reactive oxygen species in Drosophila. Genes Cells 2020, 25, 343-349. [CrossRef] [PubMed]

57. Das, M.; Garlick, D.S.; Greiner, D.L.; Davis, R.J. The role of JNK in the development of hepatocellular carcinoma. Gene Dev. 2011, 25, 634-645. [CrossRef]

58. Ahmed-de-Prado, S.; Diaz-Garcia, S.; Baonza, A. JNK and JAK/STAT signalling are required for inducing loss of cell fate specification during imaginal wing discs regeneration in Drosophila melanogaster. Dev. Biol. 2018, 441, 31-41. [CrossRef]

59. Tian, A.G.; Shi, Q.; Jiang, A.; Li, S.X.; Wang, B.; Jiang, J. Injury-stimulated Hedgehog signaling promotes regenerative proliferation of Drosophila intestinal stem cells. J. Cell Biol. 2015, 208, 807-819. [CrossRef]

60. Vancamelbeke, M.; Vermeire, S. The intestinal barrier: A fundamental role in health and disease. Expert Rev. Gastroenterol. Hepatol. 2017, 11, 821-834. [CrossRef]

61. Zheng, F.; Goncalves, F.M.; Abiko, Y.; Li, H.; Kumagai, Y.; Aschner, M. Redox toxicology of environmental chemicals causing oxidative stress. Redox Biol. 2020, 34, 101475. [CrossRef] [PubMed]

62. Wisidagama, D.R.; Thummel, C.S. Regulation of Drosophila intestinal stem cell proliferation by enterocyte mitochondrial pyruvate metabolism. G3 Genes Genom. Genet. 2019, 9, 3623-3630. [CrossRef] [PubMed]

63. Jiang, H.Q.; Tian, A.G.; Jiang, J. Intestinal stem cell response to injury: Lessons from Drosophila. Cell Mol. Life Sci. 2016, 73, 3337-3349. [CrossRef] [PubMed]

64. Naszai, M.; Carroll, L.R.; Cordero, J.B. Intestinal stem cell proliferation and epithelial homeostasis in the adult Drosophila midgut. Insect Biochem. Mol. Biol. 2015, 67, 9-14. [CrossRef]

65. Herrera, S.C.; Bach, E.A. JAK/STAT signaling in stem cells and regeneration: From Drosophila to vertebrates. Development 2019, 146, dev167643. [CrossRef] [PubMed]

66. Beira, J.V.; Torres, J.; Paro, R. Signalling crosstalk during early tumorigenesis in the absence of Polycomb silencing. PLoS Genet. 2018, 14, e1007187. [CrossRef]

67. Shaw, R.L.; Kohlmaier, A.; Polesello, C.; Veelken, C.; Edgar, B.A.; Tapon, N. The Hippo pathway regulates intestinal stem cell proliferation during Drosophila adult midgut regeneration. Development 2010, 137, 4147-4158. [CrossRef]

68. Santabarbara-Ruiz, P.; Lopez-Santillan, M.; Martinez-Rodriguez, I.; Binagui-Casas, A.; Perez, L.; Milan, M.; Corominas, M.; Serras, F. ROS-induced JNK and p38 signaling is required for unpaired cytokine activation during Drosophila regeneration. PLoS Genet. 2015, 11, e1005595. [CrossRef] 